\title{
ReiDoCrea, un proyecto de investigación-acción
}

\author{
ReiDoCrea, an Action-Research Project \\ José Miguel García Ramírez. Universidad de Granada \\ Recepción: 10 de marzo de 2014 | Revisión: 10 de abril de 2014 | Aceptación/Publicación: 10 de abril de 2014 \\ Evaluado por: Marzia Fiorini. Universitá di Cassino | Karen McMullin. Trent University
}

\section{Resumen}

El proyecto de innovación docente de la Universidad de Granada "ReiDoCrea" utiliza la metodología de Investigación-Acción con la intención de resolver los problemas que van surgiendo durante la implementación del proyecto. Método: Participaron 246 estudiantes $(M=27.5 ; S D=6.2)$, durante el curso académico 1012-13, elaborando artículos de investigación y contestando un cuestionario de pregunta abierta. Resultados: Se publican 34 artículos ( $n=113$ estudiantes) de 62 artículos presentados. Las categorías emergentes significativas por puntos fuertes, débiles y posibilidad de mejora (Atla.ti): Motivación $(f=90 \%)$, Satisfacción $(f=85 \%)$, Dificultad $(f=95 \%)$ y Formación $(f=99 \%)$. Conclusiones: Necesidad de implementar un programa de orientación y formación en la elaboración de un estudio de investigación.

Palabras clave: Educación | Creatividad | Innovación | Empatía | Motivación

\begin{abstract}
The ReiDoCrea innovation project of the University of Granada uses Action-Research methodology with the intention of solving problems that arises during project implementation. Method: 246 students participated in the study $(M=27.5 ; S D=6.2)$ during the academic year 1012-1013, writing research articles and answering an openquestion questionnaire. Results: 34 articles ( $n=113$ students) of 62 papers presented were published. Significant emerging categories for strengths, weaknesses and potential for improvement (Atla.ti) points: Motivation $(f=90 \%)$, Satisfaction $(f=85 \%)$, Difficulty $(f=95 \%)$ and Training $(f=99 \%)$. Conclusions: Need to implement an orientation and training program in the development of a research study.
\end{abstract}

Keywords: Education | Creativity | Innovation | Empathy | Motivation

La sociedad reclama una enseñanza renovada, creativa, interdisciplinar y contextualizada. A través del uso de las Tecnologías de la Información y la Comunicación, TIC, el proyecto de innovación docente de la Universidad de Granada "ReiDoCrea" consolida su revista electrónica de investigación en la que los estudiantes publican los estudios de investigación que realizan durante el curso académico. Se utiliza la metodología de Investigación-Acción, porque permite abordar y resolver los problemas que surgen de manera inmediata; así se asegura el cumplimiento de los objetivos marcados por el proyecto: Mejorar la docencia, como proceso de enseñanzaaprendizaje; incrementar la satisfacción y la motivación en docentes y estudiantes; aumentar la motivación en la educación universitaria; desarrollar un nuevo instrumento de evaluación del rendimiento académico; y divulgar el conocimiento adquirido. (DíazMarín, Vázquez-Martínez \& McMullin, 2014; Fiorini y García-Ramírez, 2013; GarcíaRamírez, 2014, 2013, 2012, 2011; Herrero, 2012). 


\section{Método}

\section{Participantes}

Participaron 246 estudiantes, 163 mujeres y 83 hombres, con edades comprendidas entre 20 y 61 años $(M=27.5, S D=6.2)$. Los participantes participaron voluntariamente y eran estudiantes de las titulaciones o grado de Pedagogía (18\%), Primaria $(20 \%)$, Educación Social (26\%), Psicología (22\%), Logopedia (5\%) y Doctorado (9\%).

\section{Procedimiento e instrumentos}

Durante el curso académico 2012-13 los/as participantes elaboraron artículos de investigación dentro de su programa de formación. Los artículos tenían que cumplir con las normas de publicación de la revista. Después de evaluarlos, el profesorado participante los remitía a la revista para su posible publicación; luego los/as participante tenían que completar un cuestionario de una pregunta abierta en el que se le pedía que describiera su experiencia, expresando los puntos fuerte, débiles y posibilidad de mejora. Por lo tanto, se trato aplicar y evaluar el logro; entendiendo como logro la publicación del estudio de investigación. La evaluación fue por pasos de manera sistemática: $1^{\mathrm{a}}$. Evaluación positiva por el profesorado participante; $2^{\mathrm{a}}$. Valoración positiva de los trabajos por los evaluadores externos; $3^{a}$. Completar un cuestionario de pregunta abierta (máximo de 200 palabras), para explicar su experiencia en esta actividad. Los datos obtenidos se analizaron a través del programa Atla.ti para poder codificar el material y describir las categorías emergentes.

\section{Resultados}

Los/as 246 participantes presentaron 51 artículos valorados positivamente por su profesorado. El $98 \%$ de los/as participantes superaron la evaluación de las asignaturas adscritas al proyecto ReiDoCrea. De los 51 artículos, presentados en ReiDoCrea, fueron evaluados positivamente y publicados 34 , elaborados por 113 participantes, este resultado equivale al $67 \%$ de los artículos presentados.

El análisis del cuestionario para conocer los puntos fuertes, débiles y posibilidades de mejora (Atla.ti) se exponen en la siguiente tabla:

\begin{tabular}{lll} 
PUNTOS FUERTES & PUNTOS DÉBILES & POSIBILIDADES DE MEJORA \\
\hline Motivación $\mid \mathrm{f}=90 \%$ & & \\
\hline Publicación $\mid \mathrm{f}=85 \%$ & Redactar un artículo | $\mathrm{f}=95 \%$ & Formación | $\mathrm{f}=99 \%$ \\
\hline Satisfacción $\mid \mathrm{f}=85 \%$ & Revisión $\mid \mathrm{f}=65 \%$ & Feedback $\mid \mathrm{f}=66 \%$ \\
\hline
\end{tabular}

\section{Conclusiones}

Los resultados obtenidos ratifican la necesidad de potenciar la empatía y/o simpatía en la Educación Superior, con la misión de construir social o colaborativamente el conocimiento. Por lo tanto, es necesario formar, guiar, orientar y tutorizar los estudios de investigación que realizan los/as estudiantes, ya que es clave en una docencia de calidad visible. También en la Educación Superior el feedback entre estudiantes y profesorado es clave ya que está relacionado significativamente con la motivación, satisfacción y eficiencia. De esta manera se potencia el desarrollo del pensamiento creativo teniendo como base el conocimiento adquirido y contextualizado (GarcíaRamírez, 2014; Fiorini y García-Ramírez, 2013; Molina, 2012). 


\section{Referencias}

Diaz-Marin, V., Vazquez-Martinez, A.I., \& McMullin, K.J. (2014). First steps towards a university social network on personal learning environments. The International Review of Research in Open and Distance Learning, 15(3), 93-119. http://www.irrodl.org/index.php/irrodl/article/view/1679/2910

Fiorini, M.; García-Ramírez, JM. (2013). Cap. 5: Técnicas de grupo y creatividad aplicadas en el ámbito universitario,117-147. En Villena Martínez, MD.; Muñoz García, A. (2013). Recursos para la tutoría en el aula universitaria. Granada: Editorial Universidad de Granada. http://www.worldcat.org/title/recursos-para-latutoria-en-el-aula-universitaria/oclc/870115874

García-Ramírez, J.M. (2014). Selección de indicadores para la evaluación de la excelencia docente en la Universidad de Trent (Canadá). Granada: Universidad de Granada, 2014. http://hdl.handle.net/10481/30350

García-Ramírez, J.M. (2013). ReiDoCrea un proyecto de construcción y divulgación del conocimiento. Reidocrea, 2, 1-10. http://t.co/2grCZOgms7

García-Ramírez, J.M. (2012). Docencia-Creativa: una estrategia de motivación a través de la difusión del conocimiento. Reidocrea, 1, 1-3. http://hdl.handle.net/10481/33571

García-Ramírez, J.M. (2012). La comunicación, clave de excelencia visible en la Educación Superior. Journal for Educators, Teachers and Trainers, 3, 25-36. http://hdl.handle.net/10481/22300

García-Ramírez, J.M. (2012). Las Tecnologías de la Información y la Comunicación, TIC, en la educación universitaria. Andaluciaeduca, 76, 77. http://www.andaluciaeduca.com/hemeroteca/ae_digital76.pdf

García-Ramírez, J.M. (2011). Una reconsideración de la excelencia visible en la educación superior: la escucha empática. Andaluciaeduca, 66, 84. http://www.andaluciaeduca.com/hemeroteca/ae_digital66.pdf

Herrero Márquez, P. (2012). La interacción comunicativa en el proceso de enseñanzaaprendizaje. ReiDoCrea, 1, 138-143. http://hdl.handle.net/10481/21983

Molina Ramírez, A.M. (2012). Las TIC en la educación superior como vía de formación y desarrollo competencial en la sociedad del conocimiento. ReiDoCrea, 1, 106114. http://hdl.handle.net/10481/21977 